\title{
No evidence of sickness behaviour or diet selectivity in immune-challenged field crickets
}

\author{
Clint Kelly ${ }^{1}$ and Jules Mc Cabe Leroux ${ }^{1}$
}

${ }^{1}$ UQAM

May 5, 2020

\begin{abstract}
Sickness behaviour is a taxonomically-widespread coordinated set of behavioural changes that in- creases shelter-seeking while reducing levels of general activity, as well as food (anorexia) and water (adipsia) consumption, when fighting infection by pathogens and disease. The leading hypothesis ex- plaining such sickness-related shifts in behaviour is the energy conservation hypothesis. This hypothe- sis argues that sick (i.e. immune-challenged) animals reduce energetic expenditure in order have more energy to fuel an immune response, which in some vertebrates, also includes producing an energetically- expensive physiological fever. We experimentally tested the hypothesis that an immune-challenge with lipopolysaccharide (LPS) will cause Gryllus firmus field crickets to reduce their activity, increase shelter- use and avoid foods that interfere with an immune response (i.e. fat) while preferring a diet thats fuel an immune response (i.e. protein). We found little evidence of sickness behaviour in Gryllus firmus as immune-challenged individuals did not reduce their activity or increase their shelter-seeking. Neither did we observe changes in feeding or drinking behaviour nor a preference for protein or avoidance of lipids. Males tended to use shelters less than females but no other behaviours differed between the sexes. The lack of sickness behaviour in our study might reflect the fact that invertebrates do not possess energetically-expensive physiological fever as part of their immune response. Therefore, there is little reason to conserve energy via reduced activity or increased shelter use when immune-challenged.
\end{abstract}

\section{Hosted file}

sickness_behaviour_ms.pdf available at https://authorea.com/users/292308/articles/419940-noevidence-of-sickness-behaviour-or-diet-selectivity-in-immune-challenged-field-crickets 